\title{
Aspectos ecológicos do cavalo-marinho Hippocampus reidi no estuário Camurupim/Cardoso, Piauí, Brasil, fornecendo subsídios para a criação de uma Área de Proteção Integral
}

\author{
Ana Cecília Giacometti Mai ${ }^{1,3}$ \& Ierecê Maria de Lucena Rosa ${ }^{2}$ \\ ${ }^{1}$ Embrapa Meio-Norte, BR 343, Km 35, CP 341, CEP 64200-970, Parnaíba, PI, Brasil \\ ${ }^{2}$ Departamento de Sistemática e Ecologia, Universidade Federal da Paraíba - UFPB, CCEN, \\ Campus I, CEP 58059-900, João Pessoa, PB, Brasil \\ ${ }^{3}$ Autor para correspondência: Ana Cecília Giacometti Mai, e-mail: anacecilia_mai@yahoo.com.br
}

MAI, A.C.G. \& ROSA, I.M.L. Ecological aspects of the seahorse Hippocampus reidi in the Camurupim/ Cardoso estuary, Piauí State, Brazil, as subsidies for the implementation of an Environmental Protection Area. Biota Neotrop. 9(3): http://www.biotaneotropica.org.br/v9n3/en/abstract?article+bn01209032009.

\begin{abstract}
In 2005 a pool of institutions formally initiated a process via the Brazilian Environmental Agency (IBAMA) to create a full protection conservation unit encompassing the Camurupim/Cardoso and Timonha/ Ubatuba estuaries, state of Piauí, NE Brazil. Among the flagship species to generate support for the protection of those estuaries is the seahorse Hippocampus reidi, focus of the present study. The results of our one-year survey in that area revealed the occurrence of resident population of $H$. reidi, found in 19 distinct habitats and with one of the highest densities already reported for the species $\left(0.04 \mathrm{ind} / \mathrm{m}^{2}\right)$. This information highlights the crucial need to further support the protection of that area, which aside from housing H. reidi, a species listed as Data Deficient by the World Conservation Union (IUCN), is also an area of occurrence of the Leatherback Turtle (Dermochelys coriacea), species considered as Critically Endangered, and the American Manatee (Trichechus manatus manatus) a Vulnerable species, according to the IUCN Red List of Threatened Species 2008.
\end{abstract}

Keywords: conservation, protected area, NE Brazil, holdfast, density, population size, Syngnathidae.

MAI, A.C.G. \& ROSA, I.M.L. Aspectos ecológicos do cavalo-marinho Hippocampus reidi no estuário Camurupim/Cardoso, Piauí, Brasil, fornecendo subsídios para a criação de uma Área de Proteção Integral. Biota Neotrop. 9(3): http://www.biotaneotropica.org.br/v9n3/pt/abstract?article+bn01209032009.

Resumo: Em 2005, um conjunto de entidades iniciou formalmente um processo através do Instituto Brasileiro do Meio Ambiente e dos Recursos Renováveis (IBAMA) para a criação de uma unidade de conservação de proteção integral englobando os estuários Camurupim/Cardoso e Timonha/Ubatuba, no Estado do Piauí. Entre as espécies-bandeira capazes de angariar apoio para a proteção dos referidos estuários, encontra-se o cavalomarinho Hippocampus reidi, foco do presente trabalho. Os resultados do nosso estudo de um ano, na área em questão, mostraram a existência de uma população residente de $H$. reidi, encontrada em 19 habitats e com uma das mais altas densidades já reportadas para a espécie $\left(0,04 \mathrm{ind} / \mathrm{m}^{2}\right)$. Esta informação reforça a importância crucial de apoio adicional à proteção daqueles ambientes, que além de abrigar $H$. reidi, uma espécie listada como Deficiente de Dados pela União Internacional para a Conservação da Natureza (IUCN), é local de ocorrência da tataruga-de-couro (Dermochelys coriacea), espécie considerada Criticamente Ameaçada e o peixe-boi-marinho (Trichechus manatus manatus) uma espécie Vulnerável, de acordo com a Lista Vermelha das espécies ameaçadas da IUCN - 2008.

Palavras-chave: conservação, área protegida, NE do Brasil, substrato de apoio, densidade, tamanho populacional, Syngnathidae. 


\section{Introdução}

Cavalos-marinhos encontram-se fortemente ameaçados por sua grande exploração comercial e pela degradação de seus hábitats (Vincent \& Pajaro 1997, Rosa et al. 2002, Rosa et al. 2005), estando o gênero Hippocampus entre os muitos que possuem uma história de vida única, dada a sua esparsa distribuição, baixa mobilidade, pequenas áreas vitais, baixa fecundidade e longo cuidado parental (Foster \& Vincent 2004).

No Brasil, os cavalos-marinhos são comercializados em vários pontos da costa e até em localidades interioranas (Rosa et al. 2005). Seu comércio envolve desde a coleta de animais vivos para fins de aquarismo até a venda de animais secos, que são comercializados para diversas finalidades (Rosa et al. 2002, Rosa et al. 2005).

Desde o ano de 2005 encontra-se em andamento um pedido formal, junto ao Instituto Brasileiro do Meio Ambiente e Recursos Naturais Renováveis (IBAMA), para a criação de uma Unidade de Conservação de Proteção Integral englobando os estuários dos rios Camurupim/Cardoso e Timonhonha/Ubatuba (IBAMA/PARNAÍBA 2005), ambos situados na região leste do litoral do Piauí, onde este estudo foi realizado. As autoridades ambientais competentes já reconheceram a importância da região e a necessidade de criação de uma Unidade de Conservação de Proteção Integral, sugerindo a categoria de Refúgio de Vida Silvestre, porém recomendando a realização de estudos complementares e produtos cartográficos.

Esta área abriga populações de tartaruga-de-couro (Dermochelys coriacea (Linnaeus, 1766)) espécie Criticamente em Perigo (Sarti Martinez 2000), o mero (Epinephelus itajara (Lichtenstein, 1822)) espécie Criticamente em Perigo (Tak-Chuen \& Ferrera 2006), e de peixe-boi-marinho (Trichechus manatus manatus Linnaeus, 1758)), espécie Vulnerável (Deutsch et al. 2008) entre outras espécies presentes na Lista Vermelha de animais ameaçados de extinção (IUCN 2008). Constitui ainda, importante ponto de parada, alimentação e reprodução de aves costeiras residentes e migratórias do Corredor Migratório do Atlântico Ocidental (IBAMA/PARNAÍBA 2005).

Os estuários da referida região abrigam também cavalos-marinhos da espécie Hippocampus reidi Ginsburg, 1933, um peixe de grande importância comercial (Rosa 2001, Rosa et al. 2005), incluída desde 2004 no Apendice II da Lista Nacional de Espécies de Invertebrados Aquáticos e Peixes Sobreexplotadas ou Ameaçadas de Sobreexplotação, de acordo com a Instrução Normativa ${ }^{\circ} 05$, de 21 de maio de 2004, do Ministério do Meio Ambiente. Em um cenário mundial, os cavalos- marinhos estão inclusos na Lista Vermelha de animais ameaçados de extinção (IUCN 2008) e na Convention on International Trade in Endangered Species of Wild Fauna e Flora (CITES 2004).

Neste contexto, o presente trabalho teve como objetivos estudar: i) razão sexual; ii) utilização de componentes da estrutura do hábitat; iii) composição de grupos; iv) densidade; e v) tamanho populacional de $H$. reidi no estuário dos rios Camurupim/Cardoso, Piauí, para dar subsídios à implantação da unidade de conservação, bem como proporcionar as informações básicas para a determinação do status de conservação da espécie aos gestores nacionais e internacionais.

\section{Material e Métodos}

\section{1. Área de estudo}

A região de estudo compreende o estuário dos rios Camurupim/ Cardoso, situado na porção leste do Piauí (Figura 1), entre as latitudes de $02^{\circ} 53^{\prime} \mathrm{S}$ e $02^{\circ} 58^{\prime} \mathrm{S}$ e longitudes $41^{\circ} 28^{\prime} \mathrm{O}$ e $41^{\circ} 24^{\prime} \mathrm{O}$. Estes rios estão inseridos na Área de Proteção Ambiental (APA) do Delta do Parnaíba, criada pelo Decreto Federal de 28 de agosto de 1996, que envolve todo o litoral do Piauí e partes do Maranhão e Ceará.
Dentro do estuário foram selecionados cinco pontos de amostragens, dois desses no leito do Rio Camurupim e os outros três em áreas de remanso, localmente denominadas de camboas. Os pontos denominados Camurupim e Macapá estão localizados no leito do rio Camurupim. As camboas, conhecidas localmente por Canal das Garças, Camboa do Cavalo-Marinho e Jandaíra são estreitos braços do estuário onde a força da maré é minimizada pela pequena profundidade e pela presença de bancos de algas.

A área de estudo está sob a influência de marés semi-diurnas de até 3 metros de amplitude nos períodos de sigízia. O clima da região é caracterizado por temperatura média anual de $27,8^{\circ} \mathrm{C}$ e precipitação anual de 1069 mm, distribuídos de modo bastante heterogêneo. Por sua localização muito próxima ao equador, suas estações climáticas são regidas pela precipitação e caracterizadas por um período de seca (julho a dezembro) e, um período chuvoso (janeiro a junho).

\section{Métodos de amostragem}

Os dados foram obtidos através de censos subaquáticos por meio de mergulhos livres, sempre realizados diurnamente em dupla, com os dois mergulhadores procurando ativamente a espécie de interesse. As buscas ocorreram de duas formas: busca intensiva sem a medição de área percorrida e transectos de $50 \mathrm{~m}^{2}$, onde era esticada uma trena e a busca se fazia a $0,5 \mathrm{~m}$ de cada lado da trena.

Dados de temperatura da água, salinidade, transparência e amplitude da maré foram anotados a cada transecto, utilizando termômetro de álcool, refratômetro, disco de secchi e tábua de maré, respectivamente. Para cada indivíduo avistado registrou-se: sexo, altura do corpo $(\mathrm{cm})$, comportamento (se o peixe se encontrava nadando ou parado), substrato de apoio e composição de grupo.

Para a diferenciação dos sexos foram usados os seguintes parâmetros: indeterminado - indivíduos jovens que não apresentavam diferenciação sexual visível, macho - presença de bolsa incubadora e fêmea - presença de órgão ovopositor (Figura 2).

A fim de se evitar a recontagem de indivíduos, caracterizando pseudo-réplicas, e reconhecer cada cavalo-marinho encontrado, estes foram marcados individualmente por meio da combinação de seis cores de arames emborrachados e 11 cores de borrachas. Esta marcação foi posta na cauda dos indivíduos maiores que $7 \mathrm{~cm}$.

Todos os dados foram obtidos in situ e anotados em prancha de PVC, sem tirar o indivíduo da água e, tomando-se o cuidado de devolvê-lo no mesmo local onde foi encontrado.

\section{Análise de dados}

Para calcular a razão sexual foram utilizados dados dos indivíduos maiores ou iguais a $12 \mathrm{~cm}$, evitando assim a superestimação do número de fêmeas, já que jovens podem ter sido, erroneamente, classificados como fêmeas. Para verificar se houve diferença significativa entre o número de machos e fêmeas, foi realizado o teste qui-quadrado $\left(x^{2}\right)$ com correção de Yates (Martin-Smith \& Vincent 2005).

Os substratos utilizados por $H$. reidi foram identificados ao menor nível taxonômico, baseado em literatura especializada e com a ajuda de especialistas. Posteriormente, fez-se a análise da frequiência no uso dos diferentes substratos de apoio.

A análise da composição de grupos foi baseada no número e nos gêneros que compunham os agrupamentos. Indivíduos recapturados, que se apresentavam em grupo por mais de uma vez, tiveram a composição dos seus grupos comparada para a identificação de possíveis casais fiéis.

O número de avistagens por transectos foi utilizado como indicador de densidade e foi estatisticamente comparado entre os locais de coleta e entre os meses do ano, pelo teste não-paramétrico de Kruskal-Wallis (Calligari-Jacques 2003), já que os dados não se apresentaram homogêneos. Para analisar a relação entre densidade e 


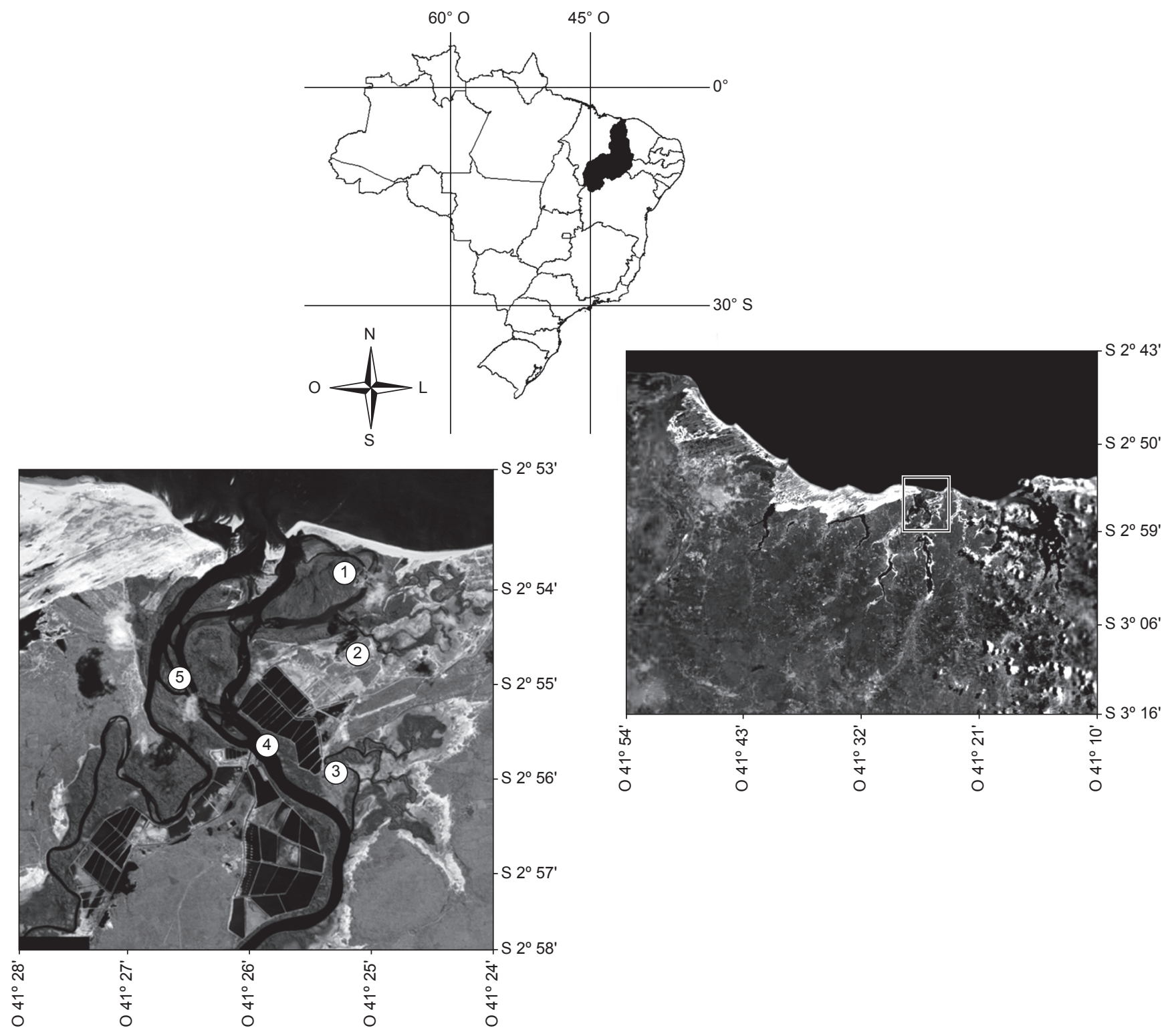

Figura 1. Litoral do Estado do Piauí, Brasil. Em detalhe o estuário Camurupim/Cardoso. Pontos de amostragem: 1) Canal da Garças; 2) Camboa do cavalomarinho; 3) Jandaíra; 4) Camurupim; e 5) Macapá. Mapa cedido pelo Instituto Chico Mendes (ICMBio).

Figure 1. Coastal zone of Piauí State, Brazil. In detail, the Camurupim/Cardoso estuary. Sampling sites: 1) Canal da Garças; 2) Camboa do cavalo-marinho; 3) Jandaíra; 4) Camurupim; and 5) Macapá. Map provided by Instituto Chico Mendes (ICMBio).

fatores abióticos (temperatura da água, salinidade, transparência) foi utilizado o teste de regressão múltipla stepwise (Zar 1996).

Os intervalos de confiança das densidades foram construídos de acordo com Buckland et al. (1993). O intervalo gerado desta maneira é sempre positivo, porém assimétrico. Todos os intervalos de confiança utilizados neste trabalho foram de $95 \%$.

O tamanho populacional foi calculado pela extrapolação dos dados de densidade para o perímetro da área coberta pelas amostragens (busca intensiva e transectos). Isto porque, os dados de densidade utilizados para esta estimativa foram baseados apenas em transectos feitos próximos a margem, sendo que o leito do estuário não teve sua densidade estimada. Devido à diferença das densidades médias por locais a estimativa do tamanho populacional foi feita para cada local em separado, sendo que o valor total é o somatório dos mesmos.

\section{Resultados}

Nos 225 transectos realizados foram avistados 449 indivíduos de $H$. reidi, enquanto outros 420 indivíduos foram encontrados por meio da busca intensiva. Retirando os indivíduos recapturados, foram considerados 647 indivíduos. A altura média dos indivíduos foi de 11,7 cm (mín. 2,6 e máx. 19 cm). A temperatura da água apresentou-se bastante homogênea no período, com média de $28,5 \pm 0,9^{\circ} \mathrm{C}$. A salinidade $(38 \pm 6,9)$ e transparência da água $(33 \pm 16,26 \mathrm{~cm})$ flutuaram marcadamente ao longo do ano (média $\pm \mathrm{DP}$ ).

\section{Razão sexual}

Dos 647 exemplares de $H$. reidi avistados, 211 (32,6\%) foram categorizados como sexo indeterminado, 233 (36\%) machos e 203 


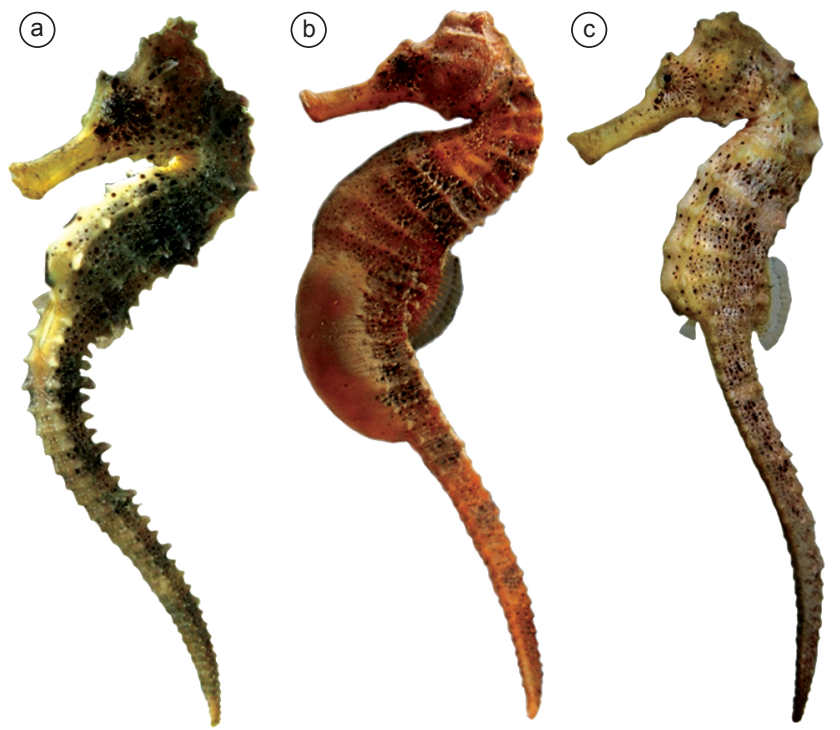

Figura 2. Hippocampus reidi: a) indivíduo de sexo indeterminado (altura $8,5 \mathrm{~cm}$ ); b) macho (altura $14 \mathrm{~cm}$ ); e c) fêmea (altura $10 \mathrm{~cm}$ ).

Figure 2. Hippocampus reidi: a) individual with undefined sex (heigth $8.5 \mathrm{~cm}$ ); b) male (heigth $14 \mathrm{~cm}$ ); and c) female (heigth $10 \mathrm{~cm}$ ).

$(31,4 \%)$ fêmeas (Tabela 1$)$. A razão sexual (número de machos pelo número de fêmeas, utilizando dados dos indivíduos maiores ou iguais a $12 \mathrm{~cm}$ ) observada foi de 1,46:1 sem diferença significativa $\left(\mathrm{x}^{2}=0,21, \mathrm{~g} .1 .=1, \mathrm{p}=0,65\right)$.

\section{Utilização de componentes da estrutura do hábitat}

Vinte substratos de apoio foram utilizados por $H$. reidi $(\mathrm{n}=548)$ no local de estudo. O principal deles foi a raiz de mangue branco (Laguncularia racemosa (Linnaeus, 1807)) com 54\% ( $\mathrm{n}=297)$ das ocorrências. Outros substratos com maior freqüência de utilização foram: mangue vermelho (Rhizophora mangle (Linnaeus, 1753) $12 \%)$, lama (11\%) e a macroalga Caulerpa sertularioides (S.G. Gmelin) M.A. Howe (8\%) (Figura 3).

Além destes, foram registrados como substrato de apoio, mas com baixa incidência $(<5 \%)$ as macroalgas Acanthophora spicifera (M. Vahl) Børgesen, Galaxaura obtusata (J. Ellis \& Solander) J.V. Lamouroux, Dyctiota sp., Ulva lactuta Linnaeus, Enteromorpha sp., Crassostrea rhizophorae (Guilding, 1828), Chondracanthus acicularis (Roth) Fredericq, Gracilaria sp. 1, 2, Hypnea sp. 1 e 2, Sargassum sp., uma alga não identificada, a fanerôgama marinha Halodule wrightii Ascherson (capim agulha), substrato artificial (pedaço de malha de rede de pesca) e esponjas.

\section{Composição de grupos}

Os cavalos-marinhos foram avistados principalmente solitários ( $83 \%$ das avistagens, $\mathrm{n}=869$ ). Entretanto, foram observados 61 agrupamentos de indivíduos sendo 45 duplas, 10 trios, três quartetos, dois quintetos e um sexteto. Dos 45 pares encontrados, 19 eram formados por macho e fêmea (42\%), sendo considerados possíveis casais reprodutivos.

Analisando indivíduos encontrados e recapturados em grupos, três pares foram considerados como casais, os quais permaneceram juntos por no mínimo 36, 48 e 63 dias. Foi registrado também um par de fêmeas que permaneceram juntas por no mínimo 64 dias. Outros oito indivíduos, apesar de terem sido recapturados em grupo, não
Tabela 1. Número de Hippocampus reidi capturados de agosto de 2006 a julho de 2007 no estuário Camurupim/Cardoso, Piauí. Ind. = indeterminado.

Table 1. Number of Hippocampus reidi captured from August 2006 to July 2007 at Camurupim/Cardoso estuary, Piauí. Ind. = undetermined.

\begin{tabular}{lrrrr}
\hline \multicolumn{1}{c}{ Mês } & Ind. & \multicolumn{1}{c}{$\hat{\boldsymbol{c}}$} & \multicolumn{1}{c}{ + } & Total \\
\hline Agosto & 14 & 46 & 28 & 88 \\
Setembro & 10 & 21 & 17 & 48 \\
Outubro & 24 & 19 & 15 & 58 \\
Novembro & 11 & 14 & 11 & 36 \\
Dezembro & 15 & 14 & 12 & 41 \\
Janeiro & 32 & 28 & 18 & 78 \\
Fevereiro & 29 & 25 & 21 & 75 \\
Março & 35 & 16 & 32 & 83 \\
Abril & 19 & 14 & 11 & 44 \\
Maio & 5 & 11 & 10 & 26 \\
Junho & 7 & 15 & 15 & 37 \\
Julho & 10 & 10 & 13 & 33 \\
Total & 211 & 233 & 203 & 647 \\
\hline
\end{tabular}

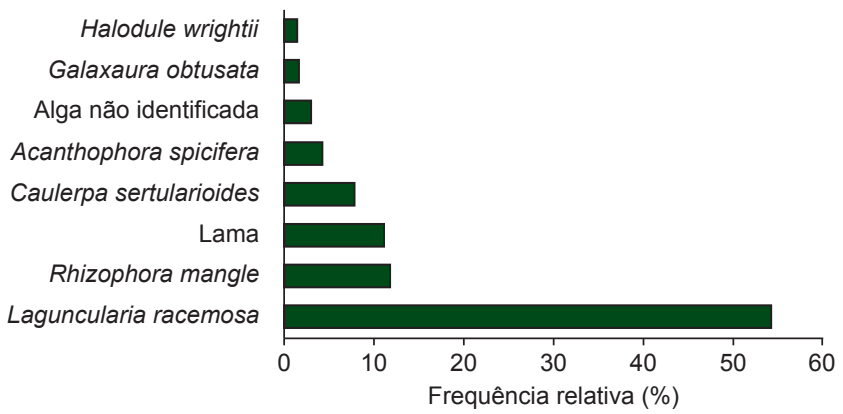

Figura 3. Freqüência no uso do substrato de apoio pelo Hippocampus reidi no período de agosto de 2006 a julho de 2007 no estuário Camurupim/ Cardoso, Piauí.

Figure 3. Frequency of holdfast use by Hippocampus reidi, from August 2006 to July 2007 at Camurupim/Cardoso estuary, Piauí.

estabeleceram duplas fiéis, sendo os grupos compostos por indivíduos não avistados anteriormente.

\section{Densidade}

A densidade média de indivíduos registrada para o estuário no período de estudo foi de $0,04 \pm 0,05 \mathrm{~m}^{-2}$, de forma que a correlação entre as densidades estimadas e os fatores abióticos mostrou-se baixa (Figura 4). A densidade variou significativamente durante os meses estudados, de 0,084 a 0,011 ind. $\mathrm{m}^{-2}\left(x^{2}=29,53\right.$, g.l. $\left.=11, \mathrm{p}=0,0019\right)$ (Figura 4b).

Espacialmente, a densidade também variou significativamente $\left(x^{2}=24,83\right.$, g.l. $\left.=4, \mathrm{p}=0,0001\right)$, com Camurupim e Jandaíra apresentando as menores densidades (0,018 e 0,019 ind. $\mathrm{m}^{-2}$, respectivamente) e Canal das Garças, Macapá e camboa do Cavalo-marinho $(0,062$, 0,059 e 0,047 ind. $\mathrm{m}^{-2}$, respectivamente) (Figura 5).

\section{Tamanho populacional}

A estimativa do tamanho populacional dado pela extrapolação dos dados de densidade média para o perímetro estudado do estuário indicou a presença de 1254 indivíduos nas áreas rasa do estuário (in- 


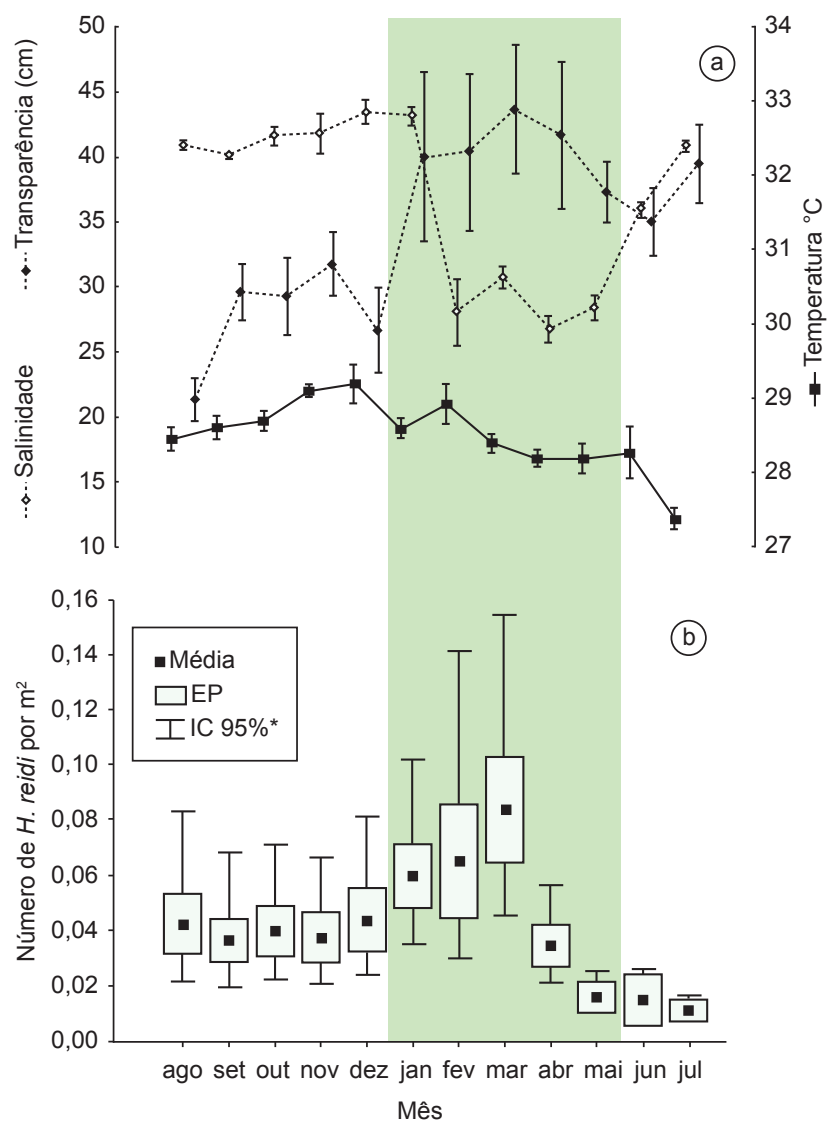

Figura 4. Variação mensal (agosto de 2006 a julho de 2007) no estuário Camurupim/Cardoso: a) média dos dados abióticos ( $\square$ temperatura da água, - transparência da água e $\diamond$ salinidade) e o erro padrão (I) de cada variável; e b) densidade de Hippocampus reidi. *Intervalo de Confiança segundo Buckland et al. (1993). Caixa cinza de janeiro a maio representando o período chuvoso.

Figure 4. Monthly variation (August 2006 to July 2007) in the Camurupim/ Cardoso estuary: a) mean values of abiotic data ( water temperature, water transparency e $\diamond$ salinity) and standart error (I) for each variable; and b) density of Hippocampus reidi. *Confidence interval follows Buckland et al. (1993). Shaded area from January to May denotes the rainfall period.

tervalo de confiança: limite inferior $=725$ e limite superior $=2182$ ) (Tabela 2).

\section{Discussão}

A criação de áreas que protejam as populações de cavalos-marinhos é um passo fundamental para a conservação de suas populações e dos seus ambientes preferenciais no Brasil, uma vez que os cavalosmarinhos são considerados excelentes espécies-bandeira (Dias \& Rosa 2003, Rosa et al. 2007). A área dos estuários Camurupim/ Cardoso e Timonha/Ubatuba é considerada crítica para conservação e criação de Unidades de Conservação por levantamentos estaduais (Aquasis 2003) e nacionais (MMA/SBF 2002).

$\mathrm{Na}$ área de estudo, indivíduos de Hippocampus reidi foram avistados ao longo do ano e a espécie reproduziu durante todo o ano, o que indica a presença de uma população residente na área de estudo. Populações naturais de cavalos-marinhos geralmente apresentam números equivalentes de machos e fêmeas adultas (Lourie et al. 1999, Foster \& Vincent 2004). O fato de não ter havido diferença
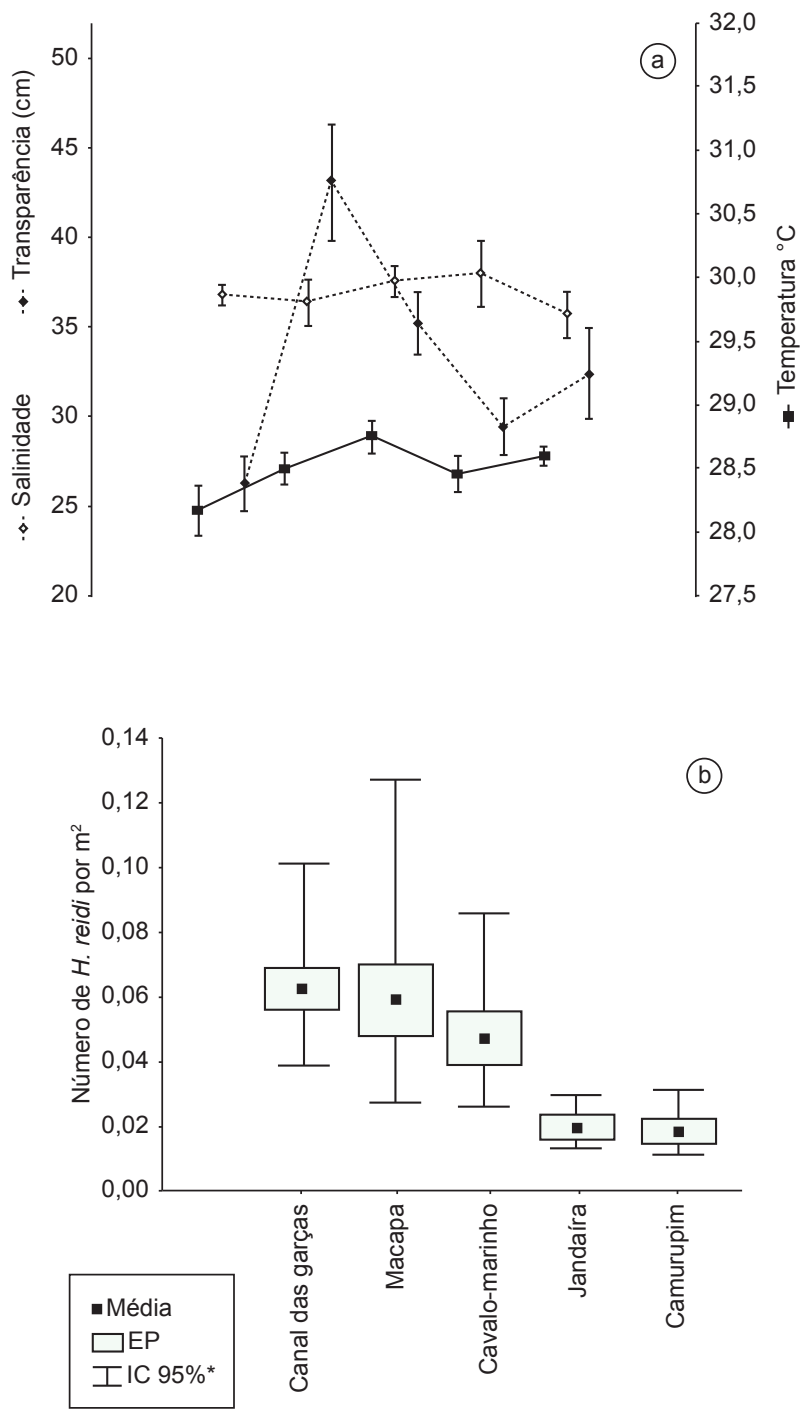

Figura 5. Variação local (agosto de 2006 a julho de 2007) no estuário Camurupim/Cardoso, Piauí: a) média dos dados abióticos ( $\boldsymbol{\square}$ temperatura, $\bullet$ transparência e $\diamond$ salinidade) e o erro padrão (I) de cada variável; e b) densidade de Hippocampus reidi. *Intervalo de Confiança segundo Buckland et al. (1993).

Figure 5. Spatial variation (August 2006 to July 2007) in the Camurupim/ Cardoso estuary: a) men values of abiotic data ( $\mathbf{w}$ water temperature, $\boldsymbol{}$ water transparency e $\diamond$ salinity) and standart error (I) for each variable; and b) density of Hippocampus reidi. *Confidence interval follows Buckland et al. (1993).

significativa na relação número de machos por número de fêmeas corrobora estudos recentes com a espécie (Rosa et al. 2007).

Nos resultados encontrados para a formação de grupos, existe concordância com trabalhos anteriores que demonstraram a formação de pares em algumas espécies de cavalos-marinhos, os quais podem durar vários eventos reprodutivos (Foster \& Vincent 2004). A reavistagem de três casais, no presente trabalho, pode ser um indicativo de um padrão monogâmico para $H$. reidi, conforme discutido em outros trabalhos, pela sua fidelidade de sítio e pequena área vital (Dias \& Rosa 2003), características biológicas de espécies de peixes monogâmicas (Vincent et al. 2005).

A observação de agrupamentos de $H$. reidi é um evento comum, já sendo relatado agrupamentos de até sete indivíduos (Rosa et al. 
Tabela 2. Estimativa do tamanho populacional de Hippocampus reidi por extrapolação para o perímetro do estuário Camurupim/Cardoso, Piauí. Sendo $\mathrm{L}_{\text {inf }}=$ limite inferior e $\mathrm{L}_{\text {sup }}=$ limite superior.

Table 2. Estimation of Hippocampus reidi population size extrapolated to perimeter of Camurupim/Cardoso estuary, Piauí. $\mathrm{L}_{\text {inf }}=$ lower limits and $\mathrm{L}_{\text {sup }}=$ upper limits.

\begin{tabular}{|c|c|c|c|c|c|}
\hline \multirow[t]{2}{*}{ Local } & \multirow{2}{*}{$\begin{array}{l}\text { Perímetro da área } \\
\text { amostrada (m) }\end{array}$} & \multirow{2}{*}{$\begin{array}{c}\text { Densidade } \\
\text { (ind.m }^{-1} \text { ) }\end{array}$} & \multirow{2}{*}{$\begin{array}{c}\text { Tamanho } \\
\text { populacional }\end{array}$} & \multicolumn{2}{|c|}{ Intervalo de confiança } \\
\hline & & & & $\mathbf{L}_{\mathrm{inf}}$ & $\mathbf{L}_{\text {sup }}$ \\
\hline Macapá & 3650 & 0,06 & 219 & 100 & 464 \\
\hline Rio Camurupim & 11698 & 0,02 & 214 & 127 & 361 \\
\hline Canal das Garças & 8643 & 0,06 & 538 & 332 & 873 \\
\hline Cavalo-marinho & 3815 & 0,05 & 179 & 98 & 326 \\
\hline Jandaíra & 5344 & 0,02 & 104 & 68 & 158 \\
\hline Total & 33150 & 0,041 & 1254 & 725 & 2182 \\
\hline
\end{tabular}

2007). O baixo número de encontros de grandes grupos segue o observado para outras espécies do gênero Hippocampus (ver Foster \& Vincent 2004).

No presente estudo, foram identificados 20 diferentes substratos de apoio utilizados por H. reidi, incluindo um artificial. O uso de estruturas artificiais foi observado em outras espécies de Hippocampus (ver Foster \& Vincent 2004), bem como em H. reidi (Dias \& Rosa 2003, Oliveira 2007) o que, por um lado, sugere uma relativa plasticidade da espécie em relação a modificações antrópicas nos ecossistemas costeiros, e por outro, torna os cavalos-marinhos mais acessíveis e vulneráveis à exploração, se fixando em piers e redes de pesca.

O uso de diversos componentes da estrutura do habitat por $H$. reidi ressalta a sua dependência do equilíbrio biológico do ecossistema, enfatizando a importância da conservação desses habitats para esta espécie.

A literatura indica que a densidade de cavalos-marinhos tende a ser baixa e que estes se distribuem em manchas (ver Foster \& Vincent 2004). Com relação a $H$. reidi, Rosa et al. (2007) demonstraram uma grande variação na densidade populacional da espécie ao longo do Brasil, de 0,0023 ind. $\mathrm{m}^{-2} \mathrm{em}$ Penha (SC) a 0,066 ind. $\mathrm{m}^{-2}$ em Tubarão (RN). O estuário Camurupim/Cardoso apresentou uma das maiores densidades de $H$. reidi já registradas $\left(0,04\right.$ ind. $\left.\mathrm{m}^{-2}\right)$ e mostrou ter uma população residente, sendo por isso, um lugar-focal para se implantar uma Área de Proteção Integral voltada para um conjunto de espécies marinho-estuarinas, dentre as quais várias ameaçadas de extinção.

A extrapolação dos dados de densidade para toda a área do estuário não pode ser realizada por dois motivos: $H$. reidi tem distribuição agregada (Dias \& Rosa 2003) e indefinição da área estuarina, devido aos poucos estudos realizados na região. Sendo assim, o valor encontrado de 1254 indivíduos nas áreas rasas do estuário é subestimado, porém muito relevante devido a inexistência de dados de tamanho populacional para a espécie.

\section{Agravantes para criação de uma Área de Proteção Integral}

É crescente a implantação de grandes empreendimentos turísticos na região, tais como: hotéis, resort, revitalização do aeroporto internacional de Parnaíba/PI e pavimentação de diversas vias de acesso (IBAMA/PARNAÍBA 2005).

Outro impacto ambiental vem sendo causado pela atividade da carcinicultura, que degrada e suprime manguezais, além de desestruturar comunidades tradicionais (IBAMA/PARNAÍBA 2005). A implantação de projetos de engorda de camarão exótico em cativeiro (Litopennaeus vannamei (Boone,1931)) na região já vem ocupando
Áreas de Preservação Permanente, como margens de rios, manguezais e ambientes intertidais associados.

Nesta região, H. reidi foi alvo de elevado esforço de pesca para ser comercializada para aquarismo (Rosa et al. 2005), havendo também comércio de exemplares secos para fins ornamentais. Até hoje é comum sua pesca para uso na medicina tradicional e as capturas incidentais, com impactos populacionais ainda não estimados.

Cabe destacar que, atualmente, vem se explorando de forma ainda não disciplinada, o turismo ecológico na região, onde o cavalo-marinho é o principal atrativo. Com a criação de uma Área de Proteção Integral, algumas espécies presentes na área teriam investimentos diretos em pesquisas, e trabalhos de educação ambiental e ordenamento do turismo seriam efetivamente realizados. As demais espécies, que utilizam o estuário Camurupim/Cardoso e Timonha/ Ubatuba, se beneficiariam da preservação desse ecossistema, como por exemplo, os peixes e aves que utilizam estes estuários para se reproduzir e se alimentar.

\section{Agradecimentos}

A PADI-Fundation por financiar parte das pesquisas, a Capes pela bolsa de estudos da primeira autora. Ao ICMBio pela licença de coleta e apoio logístico. Ao Dr. Ricardo Rosa, Dr. Gonzalo Velasco, Dr. Daniel Loebmann, Dr. Alexandre M. Garcia e Dra. Mônica G. Mai pelas contribuições e correções.

\section{Referências Bibliográficas}

AQUASIS. 2003. A Zona costeira do Ceará: diagnóstico para a gestão integrada. Aquasis, Fortaleza, p. 1-293.

BUCKLAND, S.T., ANDERSON, D.R., BURNHAM, K.P. \& LAAKE, J.L. 1993. Distance sampling: estimating abundance of biological populations. Chapman \& Hall, London, p. 446.

CALLIGARI-JACQUES, S.M. 2003. Bioestatística: princípios e aplicações. Artmed Editora SA, Porto Alegre, p. 256.

Convention on International Trade in Endangered Species of wild Fauna and Flora - CITES. 2004. Meeting of the nomenclature Committee Geneva (Switzerland). Project Seahorse, Columbia, p. 1-5.

DEUTSCH, C.J., SELF-SULLIVAN, C. \& MIGNUCCI-GIANNONI, A. 2008. Trichechus manatus. In IUCN Red list of threatened species. www. iucnredlist.org (último acesso em 03/05/2009).

DIAS, T.L. \& ROSA, I.L. 2003. Haitat preferences of a seahorse species, Hippocampus reidi (Teleostei: Syngnathidae) in Brazil. Aqua J. Ichthyol. Aquat. Biol. 6(4):165-176.

FOSTER, S. \& VINCENT, A.C.J. 2004. The life history and ecology of seahorses, Hippocampus spp.: implications for conservation and management. J. Fish Biol. 65:1-61. 
Instituto Brasileiro do Meio Ambiente e dos Recursos Naturais Renováveis IBAMA. 2005. Proposta de Criação de uma unidade de conservação de proteção integral na zona costeira dos Estados do Piauí e Ceará. IBAMA, Parnaíba, p. 1-32.

International Union for Conservation of Nature - IUCN. 2007. Red list of threatened species. Cambridge. www.iucnredlist.org (último acesso em 04/06/2008)

LOURIE, S.A., VINCENT, A.C.J. \& HALL, H.J. 1999. Seahorses: an identification guide to the world's species and their conservation. Project Seahorse, London, p. 1-224.

MARTIN-SMITH, K.M. \& VINCENT, A.C.J. 2005. Seahorse declines in the Derwent estuary, Tasmania in the absence of fishing pressure. Biol. Conserv. 123:533-545.

Ministério do Meio Ambiente- MMA. 2002. Avaliação e identificação de áreas e ações prioritárias para a conservação, utilização sustentável e repartição dos benefícios da biodiversidade nos biomas brasileiros. MMA, Brasília, p. 1-404.

OLIVEIRA, T.P.R. 2007. Ecologia populacional de Hippocampus reidi (Teleostei: Syngnathidae) em dois estuários do estado de Pernambuco, Brasil. Dissertação de Mestrado, Universidade Federal da Paraíba, João Pessoa, p. 69.

ROSA, I.L. 2001. Captura ameaça cavalos-marinhos. Jornal do Comércio, Ciência e Meio Ambiente, Recife.

ROSA, I.L., DIAS, T.L. \& BAUM, J.K. 2002. Threatened fishes of the world: Hippocampus reidi Ginsburg, 1933 (Syngnathidae). Environ. Biol. Fishes. 64(4):378.

ROSA, I.M.L., ALVES, R.R.N., BONIFÁCIO, K.M., MOURÃO, J.S., OSÓRIO, F.M., OLIVEIRA, T.P.R. \& NOTTINGHAM, M.C. 2005.
Fishers' knowledge and seahorse conservation in Brazil. J. Ethnobiol. Ethnomed. 1:1-15.

ROSA, I.M.L., OLIVEIRA, T.P.R., CASTRO, A.L.C., MORAES, L.E.S., XAVIER, J.H.A., NOTTINGHAM, M.C., DIAS, T.L.P., BRUTO-COSTA, L.V., ARAÚJO, M.E., BIROLO, A. B., MAI, A.C.G. \& MONTEIRO-NETO, C. 2007. Population characteristics, space use and habitat associations of the seahorse Hippocampus reidi Ginsburg, 1933. Neotrop. Ichthyol. 5(3):405-414.

SARTI MARTINEZ, A.L. 2000. Dermochelys coriacea. In IUCN Red list of threatened species. www.iucnredlist.org (último acesso em 03/05/2009).

TAK-CHUEN, T.C. \& FERRERA, B.P. 2006. Epinephelus itajara. In IUCN Red list of threatened species. www.iucnredlist.org (último acesso em 03/05/2009).

VINCENT, A.C.J. \& PAJARO, M.G. 1997. Community-based management for a sustainable seahorse fishery. In Proceedings of the 2 World Fisheries Congress. CSIRO, Australia, p. 761-766.

VINCENT, A.C.J., EVANS, K.L. \& MARSDEN, A.D. 2005. Home range behaviour of the monogamous Australian seahorse, Hippocampus whitei. Environ. Biol. Fishes. 72(1):1-12.

ZAR, J.H. 1996. Biostatistical analysis. 3 ed. Prentice-Hall International, New Jersey, p. 662.

Recebido em 03/06/09

Versão reformulada recebida em 04/06/09

Publicado em 11/06/09 